\title{
Pre-ovulatory intercellular regulation of miR-125a-3p within mouse ovarian follicles
}

\author{
Hadas Grossman*, Efrat Har-Paz*, Natalie Gindi, Irit Miller and Ruth Shalgi \\ Department of Cell and Developmental Biology, Sackler Faculty of Medicine, Tel-Aviv University, Ramat-Aviv, \\ Tel-Aviv, Israel
}

Correspondence should be addressed to R Shalgi; Email: shalgir@tauex.tau.ac.il

*(H Grossman and E Har-Paz contributed equally to this work)

\begin{abstract}
miR-125a-3p, a post-transcription regulator of Fyn kinase, is expressed in mouse pre-ovulatory follicles; its expression within the follicle decreases toward ovulation. Our aim was to follow the synthesis of miR-125a-3p and regulation of its expression in all follicular compartments, focusing on intercellular communication. Mural granulosa cells (GCs) or cumulus cells (CCs) were transfected with either scrambled-miR (negative control) or miR-125a-3p mimic. Freshly isolated GCs or CCs were incubated overnight in culture media conditioned by transfected cells. To examine a possible role of gap junctions in the regulation of miR$125 a-3 p$, we incubated large antral follicles in the presence of carbenoxolone, a gap-junction inhibitor, and triggered them to mature with hGC. Levels of miR-125a family members in GCs, CCs, oocytes, and culture media were measured by qPCR. We showed that miR-125a-3p is synthesized by all follicular components, but is regulated within the follicle as a whole. It is secreted by mural-GCs and taken up by CCs, where it remains functional, and vice versa, mural-GCs can take up miR-125a-3p secreted by CCs. miR-125a-3p is transcribed and accumulated in oocytes throughout oogenesis. Transcriptionally quiescent GV oocytes utilize their accompanying follicular cells to monitor the level of miR-125a-3p within them, as indicated in an ex vivo follicle culture. Our study reveals that miR-125a-3p expression is modulated by a network of intercellular communications within pre-ovulatory follicles, thus enabling a coordinated decrease of miR-125a-3p toward ovulation.

Reproduction (2020) 159 215-225
\end{abstract}

\section{Introduction}

Mammalian oocytes enter the first meiotic division during embryonic life, arrest around the time of birth at the first meiotic prophase and characterized by a prominent nucleus, the germinal vesicle (GV). At this time, oocytes are enclosed by flattened somatic pregranulosa cells, forming the primordial follicles (Pepling 2012), which gradually grow and develop into primary and secondary follicles at the appropriate time. Formation of an antrum, filled with follicular fluid (FF), defines two populations of differentiated granulosa cells facing different fates. The cumulus oophorous cells which are in direct contact with the oocyte will continue to escort the ovulated oocyte, whereas the mural granulosa cells (GCs), together with the theca cells, will form the corpus luteum after ovulation (Smitz \& Cortvrindt 2002).

Folliculogenesis and ovulation of a mature fertilizable oocyte are dependent upon the ability of all the follicular components to maintain a network of intercellular communication. These interactions are essential for providing metabolic support for the oocyte and for synchronizing the growth of the follicle with that of its enclosed oocyte (Kidder \& Mhawi 2002,
Canipari et al. 2012). The intercellular communication is particularly important toward ovulation, when multiple molecular signaling pathways within selected large antral follicles are recruited in a synchronized manner, in response to the LH surge, to support oocyte maturation, cumulus cell expansion, follicular wall rupture, and mural GCs luteinization (Sun et al. 2009). The antral follicles employ several means of autocrine and paracrine intercellular communications through the follicular fluid and maintain networks of gap junctions between GCs and within cumulus-oocyte complexes (Kidder \& Mhawi 2002, Conti et al. 2016). Komatsu and Masubuchi demonstrated recently that the oocyte membrane, at all stages of development, fuses with the membranes of GCs to form a large complex that enables transfer of large molecules (Komatsu \& Masubuchi 2018).

miRNAs, a group of naturally occurring non-coding RNAs, are small molecules that can be transferred between cells (Bartel \& Chen 2004). They are initially transcribed in the nucleus by RNA polymerase II to form a large primary miRNA (pri-miRNA), which is then processed to a stem and loop precursor miRNA (premiRNA) and transported to the cytoplasm. 
miRNAs enclosed within extracellular vesicles or in a vesicle-independent form (Mittelbrunn \& SánchezMadrid 2012, Machtinger et al. 2016) are present in body fluids (Cheng 2015), including blood (Chen et al. 2008), saliva (Michael et al. 2010), follicular fluid (da Silveira et al. 2012), urine (Lorenzen et al. 2011), and breast milk (Hata et al. 2010). miRNAs can also be transferred through gap junctions, which allow more specific targeting of recipient cells (Lemcke et al. 2015). Several miRNAs have been detected in human, equine, and bovine FFs (Di Pietro 2016). The expression pattern of miRNAs in the FF is hormonally dependent (Noferesti et al. 2015), associated with the ovulation potential of follicles (Donadeu \& Schauer 2013) and the competence of oocyte maturation (Moreno et al. 2015).

Fyn kinase is known to be involved in oocyte maturation and the first cleave (Levi et al. 2010a,b). One of the miRNAs found in human FFs is miR-125a-3p (Sang et al. 2013), which is derived from the $3 p$ arm of the premiR-125a. Our previous studies, both in HEK-293T cells (Ninio-Many et al. 2013) and in rodent GCs (Grossman et al. 2015), have established miR-125a-3p as a posttranscription regulator of Fyn. The expression of miR$125 a-3 p$ decreases in mouse oocytes during maturation; overexpression of miR-125a-3p inhibits resumption of the first meiotic division, through modulation of Fyn expression (Grossman et al. 2017). The preovulatory expression of miR-125a-3p in mural GCs was downregulated by human-chorionic gonadotropin (hCG; an $\mathrm{LH}$ analog), enabling the increase in the expression of Fyn and promoting the migratory ability of the cells; both required for proper ovulation (Grossman et al. 2015). It is largely accepted that the common receptor for LH/hCG (LHCGR) is expressed mainly by mural GCs, which are thought to transduce the ovulatory stimulus to their enclosed cumulus-oocyte complex. Because the alteration in miR-125a-3p expression within the oocytes occurs downstream to the ovulatory signal of LH/hCG, we postulate that the expression of miR-125a-3p within the follicle toward ovulation may be regulated through the intercellular communication networks between mural GCs, CCs, and the oocyte.

Here, we demonstrate that miR-125a-3p, secreted by mural GCs, can be taken up by CCs and remain functional. hCG can directly downregulate the expression of miR$125 a-3 p$ in CCs as well. Following the closing of the gap junctions within large antral follicles, the pri-miR-125a is processed to pre-miR125 and then to the mature miR125a-3p and miR-125a-5p. Exposing the follicles to hCG in vitro resulted in a decrease of miR-125a-3p expression, suggesting that the effect of hCG on miR-125a-3p within oocytes is not mediated solely by gap junctions.

Taken together, our results indicate that though all follicular components are capable of transcribing miR$125 a-3 p$, it is regulated in the pre-ovulatory follicle as a whole, utilizing means of intercellular communication. This unique type of regulation may enable the rapid synchronized changes taking place within the follicle toward ovulation.

\section{Materials and methods}

\section{Animals}

ICR female mice (Envigo RMS Limited, Jerusalem, Israel) were housed in temperature- and humidity-controlled rooms at the animal facility of the Sackler Faculty of Medicine, Tel Aviv University, under artificial illumination for $12 \mathrm{~h}$ daily. Food and water were provided ad libitum. Animal care and all experiments were in accordance with the guidelines and approval of the Institutional Animal Care and Use Committee, Sackler faculty of Medicine, Tel-Aviv University (permit No. M-14-003).

\section{Isolation of oocytes}

\section{Growing oocytes}

Growing oocytes were isolated from ovaries of 10-12 day old mice, according to an established method (Heller et al. 1981). The majority of follicles in these mice are pre-antral and contain transcribing GV oocytes (Tan et al. 2009, Ma et al. 2013). Ovaries were excised into M2 medium (M-7167; Sigma-Aldrich) supplemented with 5\% fetal bovine serum (FBS; Biological Industries, Beit-Ha'emek, Israel), penicillin, streptomycin (Biological Industries), $4 \mathrm{mg} / \mathrm{mL}$ collagenase type IV (C5138; Sigma-Aldrich), 1\% trypsin EDTA solution B (03052-1A; Biological Industries), and $5 \mathrm{mg} / \mathrm{mL}$ DNAse (D5025; Sigma Aldrich). Ovaries were pipetted every 8-10 min until the denuded oocytes could be harvested.

\section{Fully grown GV oocytes}

Fully grown GV oocytes were isolated from ovaries of 24-dayold mice, $48 \mathrm{~h}$ after s.c. administration of 5IU pregnant mare serum gonadotropin (PMSG; SyncroPart, Sanofi, Paris, France). Oocytes were isolated by gently puncturing the ovaries in Leibovitz (L)-15 culture medium (L-1518; Sigma-Aldrich) supplemented with $10 \%$ FBS, penicillin, streptomycin, and 1 $\mu \mathrm{M}$ milrinone (M-4659; Sigma-Aldrich) to prevent spontaneous maturation of the oocytes.

\section{Oocyte fixation and staining}

Oocytes were fixed in $4 \%$ paraformaldehyde devoid of methanol (Thermo Fisher Scientific) and the zonae pellucidae of fully grown GV oocytes were removed by acidic Tyrode's solution (Sigma-Aldrich). All oocytes were permeabilized using $0.005 \%$ Nonidet P (NP) - 40 dissolved in PBS containing $0.03 \%$ FBS, and stained with Hoechst (32242; Sigma-Aldrich). Oocytes were visualized and photographed by a Leica laser confocal microscope (SP8; Leica Microsystems).

\section{Ex vivo culture of antral pre-ovulatory follicles}

Pre-ovulatory follicles were cultured as described previously (Sela-Abramovich et al. 2006). Briefly, ovaries of 24-day-old mice primed with 5 IU PMSG for $48 \mathrm{~h}$ were excised into L-15 
culture medium supplemented with 10\% FBS, $1 \mu \mathrm{M}$ milrinone, penicillin, and streptomycin. Large antral follicles were mechanically isolated under a Nikon ECLIPSE Ti microscope (Nikon Instruments). Isolated follicles were incubated in $25 \mathrm{~mL}$ Erlenmeyer flasks containing $2 \mathrm{~mL}$ of DMEM/F-12 (Biological Industries) medium supplemented with $10 \%$ FBS, penicillin, streptomycin, $1 \mu \mathrm{M}$ milrinone and $100 \mu \mathrm{M}, 1 \mathrm{IU} / \mathrm{mL}$ of hCG, or Carbenoxolone (CBX; gap-junction inhibitor; C4790; SigmaAldrich), or an equal volume of vehicle. Flasks were gassed for 2 min with $\mathrm{O}_{2}: \mathrm{N}_{2}(1: 1)$ before 4 -h incubation at $37^{\circ} \mathrm{C}$ with constant shaking. In some experiments, follicles were cultured for $4 \mathrm{~h}$ with or without $100 \mu \mathrm{M} \mathrm{CBX}$; a concentration sufficient to induce GVBD ((Sela-Abramovich et al. 2006); validated by us, data not shown), in the presence of milrinone, to prevent spontaneous resumption of meiosis.

\section{Harvest of primary GCs and CCs}

Mural GCs and CCs were isolated from pre-ovulatory follicles of 24-day-old mice, $48 \mathrm{~h}$ after administration of 5 IU PMSG. Ovaries were excised into L-15 medium supplemented with $10 \%$ FBS, penicillin, and streptomycin and the large antral follicles were gently punctured to allow the release of the cumulus-oocyte complexes (COCs). The COCs were then transferred to a new plate and the CCs were mechanically detached from their enclosed oocytes. After removal of the COCs, the follicle remnants were further punctured to allow the mural GCs to ooze out. Mural GCs and CCs were collected, centrifuged at $300 \mathrm{~g}$ for $5 \mathrm{~min}$ at room temperature and either lysed or re-suspended in DMEM/F-12 medium, supplemented with $10 \%$ FBS.

\section{Transfection of mural GCs and CCs}

Harvested mural GCs and CCs were incubated for $1 \mathrm{~h}$ to allow adherence. Cells were transfected with a miR-125a-3p mimic (20 nM, MC12378; Thermo Fisher Scientific) or with 20 nM scramble-miR (4464059; Thermo Fisher Scientific) using Lipofectamine 2000 transfection reagent (Thermo Fisher Scientific) and Opti-MEM medium (Thermo Fisher Scientific), according to the manufacturer's instructions. After $4 \mathrm{~h}$ of incubation, the cells were washed three times and incubated overnight in a fresh medium before being subjected to further procedures (conditioned media, determination of the $\mathrm{miR}$ family member's level).

\section{RNA isolation, reverse transcription and quantitative $P C R$}

\section{Oocytes}

For each experiment, an equal number of oocytes were lysed and RNA was extracted using the TaqMan® MicroRNA Cellsto-CT'M kit (4391848; Thermo Fisher Scientific) according to manufacturer's instructions. RNA was reverse transcribed using the High-Capacity cDNA RT kit (4374966; Applied Biosystems). For detection of pri-miR-125a, total RNA was reverse transcribed from $9 \mu \mathrm{L}$ of the cell-to-CT lysate $(45 \%$ of the reaction volume), using random primers. Mature miRNAs were reverse transcribed from $5 \mu \mathrm{L}$ of the Cell-to-CT lysate (33\% of the reaction volume), using specific primers. Changes in the expression levels of pri-miR-125a and mature miRNAs were detected by the TaqMan ${ }^{\circledR}$ Universal PCR Master Mix (4304437; Applied Biosystems), using $2.7 \mu \mathrm{L}$ cDNA and specific primers: miR-125a-3p (assay ID 002199; Thermo Fisher Scientific), miR-125a-5p (assay ID 002198; Thermo Fisher Scientific), pri-miR-125a (assay ID AB4427012; Thermo Fisher Scientific), with a U6 snRNA (assay ID 001973; Thermo Fisher Scientific), serving as endogenous control for both primiR-125a and mature miRNAs.

\section{Mural GCs, CCs and whole ovaries}

Total RNA was extracted using TRIzol reagent (Thermo Fisher Scientific). Lysates were reverse transcribed using the HighCapacity cDNA RT Kit (4374966; Applied Biosystems) and random primers for the detection of mRNAs and pri-miR-125a, or specific primers for the detection of mature miRNAs. Changes in the level of mRNAs expression were detected by SYBR green reagent (4344463; SYBR Green PCR Master Mix; Thermo Fisher Scientific), using $20 \mathrm{ng}$ CDNA and the following primers: Fyn (mouse), forward primer 5'-ACCTCCATCCCGAACTACAAC-3' and reverse primer 5'-CATAAAGCGCCACAAACAGTG-3', hypoxanthine phosphoribosyltransferase 1 (Hprt1; mouse) forward primer 5'-CTCATGGACTGATTATGGACAGGA-3' and reverse primer 5'-GCAGGTCAGCAAAGAACTTATAGCC-3', and Lhcgr (mouse), forward primer 5'-AACCTCCCTCGGTTAAAATACCT-3' and reverse primer 5 '-CCCTTGGAAAGCGTTCCCT-3'. Changes in the expression levels of pri-miR-125a and mature miRNAs were detected by the TaqMan ${ }^{\circledR}$ Universal PCR Master Mix (4304437; Applied Biosystems), using $20 \mathrm{ng}$ cDNA and the listed specific primers.

\section{Conditioned media}

Cell media were collected and centrifuged at $300 \mathrm{~g}$ for $5 \mathrm{~min}$ at $25^{\circ} \mathrm{C}$ to remove cell debris. RNA was isolated from a total of $200 \mu \mathrm{L}$ medium, supplemented with $20 \mathrm{fmol} / \mu \mathrm{L}$ synthetic cel-miR-39-3p (VC40001; Sigma-Aldrich), serving as a control for changes in RNA occurring during purification. We used the mirVana ${ }^{\text {TM }}$ PARISTM RNA and Native Protein Purification kit (AM1556; Ambion), according to manufacturer's instructions. RNA was reverse transcribed using the High-Capacity cDNA RT kit (4374966; Applied Biosystems). Changes in the expression levels of mature miRNAs were detected by the TaqMan® Universal PCR Master Mix (4304437; Applied Biosystems), using $20 \mathrm{ng}$ CDNA and the specific primers for miR-125a-3p (assay ID 002199; Thermo Fisher Scientific), and U6 snRNA (assay ID 001973; Thermo Fisher Scientific), with cel-miR-39-3p (assay ID 000200; Thermo Fisher Scientific), used for normalization. The ABI Prism 7900 Sequence PCR machine (Applied Biosystems) was used for all procedures.

\section{XTT assay}

XTT test (Biological Industries) was performed on treated cells according to manufacturer's protocol. In short, XTT reaction solution $(50 \mu \mathrm{L})$ was added to each well and the cells were incubated for $2-4 \mathrm{~h}$ at $37^{\circ} \mathrm{C}$. Resulting color was measured by 
the SpectraMax 190 microplate reader (Molecular Probes) at a wavelength of $450 \mathrm{~nm}$ against a background control.

\section{Statistical analysis}

Experiments were repeated independently three times. Unless otherwise mentioned, the values in each experiment were normalized to the matching control. In all instances where raw data were not normally distributed, log transformation was applied to normalize the data. Normally distributed data were evaluated by one-sample Student's $t$-test. Data are expressed as mean \pm S.E.M. $P<0.05$ was considered statistically significant.

\section{Results}

\section{Expression of miR-125a family members in CCs and in mural GCs}

As mentioned in the introduction, miRNAs are initially transcribed in the nucleus as pri-miRNA and then processed to pre-miRNA. A single molecule of premiRNA can theoretically give rise to two mature miRNAs, derived from either the 5 prime $(5 p)$ or the 3 prime (3p) arms of the pre-miRNA, both capable of silencing target mRNAs (Su et al. 2009). We, thus, examined the levels of miR-125a family members in CCs and in mural GCs isolated from large follicles and subjected them to qPCR analysis for detection of pri-miR-125a, miR$125 a-3 p$, and miR-125a-5p. Normalizing the levels of miR-125a-3p and miR-125a-5p to that of pri-miR-125a revealed a similar miR-125a-3p to miR-125a-5p ratio in both CCs and mural GCs, though, miR-125a-5p is by far the predominant form (Table 1).

\section{Mural GCs can regulate the level of miR-125a-3p within CCs via the culture medium}

Because miRNAs may be used by cells as intercellular signal molecules, we examined whether miR$125 a-3 p$, secreted by mural GCs, could modulate the communication between mural GCs and CCs. Due to relatively low level of endogenous miR-125a-3p and the limited amount of mural GCs and CCs, this set of experiments was performed in transfected cells. Mural GCs were transfected with either miR-125a-3p mimic or scramble-miR as control, and then washed thoroughly and incubated overnight in fresh culture medium. We confirmed that transfected GCs overexpress miR-125a-3p (Fig. 1A) and increase its secretion into the culture medium (Fig. 1B). Based on earlier studies (Sugiura et al. 2010, Yang et al. 2016), we incubated freshly isolated CCs in conditioned medium of mural GCs transfected with either miR-125a-3p mimic or scramble-miR, and then measured in them the uptake of miR-125a-3p. The level of miR-125a-3p in CCs was higher when GCs were transfected with miR-125a-3p mimic than in CCs incubated in culture medium of mural GCs transfected with scramble-miR (Fig. 1C), suggesting that CCs can take up miR-125a-3p secreted by mural GCs. To validate that the differences in miR-125a-3p levels resulted from the transfection, we examined the level of pri-miR-125a in the nuclei of recipient CCs. CCs expressed similar levels of pri-miR-125a, regardless of miR content in the incubation medium, whether it was miR-125a-3p mimic or scrambled miR (Fig. 1D), indicating that the differences in the levels of miR-125a-3p are probably not the result of processing of the induced pri-miR-125a. To validate that miR-125a-3p remains functional within recipient CCs, we measured the expression level of Fyn. Because just a small amount of recipient CCs was available, we measured only the level of Fyn mRNA and not the level of FYN protein. Fyn mRNA, a direct downstream target of miR-125a-3p, is degraded in several cell lines upon overexpression of miR-125a-3p, in correlation with a decrease in FYN protein expression (Ninio-Many et al. 2013, Grossman et al. 2015). Here we showed that the expression of Fyn mRNA was significantly lower in CCs incubated in culture media conditioned by miR-125a-3p mimic-transfected mural GCs than in those incubated in culture media conditioned by scramble-miR-transfected GCs (Fig. 1E); indicating that miR-125a-3p, taken up by CCs, remains functional. To confirm that miR-125a-3p does not affect cell viability, mural GCs and CCs were transfected with scramble miR or miR-125a-3p mimic, culture media were replaced with fresh ones after four hours, for an overnight incubation of cells. Cells were subjected to XTT Cell Proliferation Assay for measurement of cellular necrosis or apoptosis, according to manufacturer's instructions. Absorbance (at 450-500 $\mathrm{nm}$ and $630-690 \mathrm{~nm}$ ) that was measured every hour for a period of $5 \mathrm{~h}$, for each cell type (mural GCs or CCs) and for each treatment (Scr or miR-125a-3p). In three independent experiments, no difference was observed between the treatments, indicating that the changes in miR-125a-3p levels in the culture media were not due to compromised viability of miR-125a-3p overexpressing cells (data not shown).

Table 1 Relative expression of miR-125a family members.

\begin{tabular}{lccccc}
\hline & pri-miR-125a & miR-125a-3p & miR-125a-5p & miR-125a-3p/miR-125a-5p ratio & $\boldsymbol{t}$-test $\mathbf{m i R - 1 2 5 a - 3 p / m i R - 1 2 5 a - 5 p}$ \\
\hline CCs & 1 & 0.03 & 1.50 & 0.02 & $5.6 \mathrm{E}-07$ \\
Mural & 1 & 0.09 & 1.76 & 0.05 & 0.032 \\
GCs & & & & &
\end{tabular}

Relative expression was measured in CCs and in mural GCs isolated from large follicles. Results were normalized to the level of pri-miR-125a. Data were analyzed by one-tail one-sample Student $t$-test. Values are mean \pm S.E.M. of at least three independent experiments. $* P<0.05$. 
A

A miR-125a-3p expression in transfected mural GCs

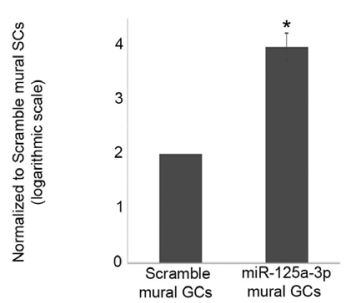

pri-miR-125a expression in recipient cumulus cells

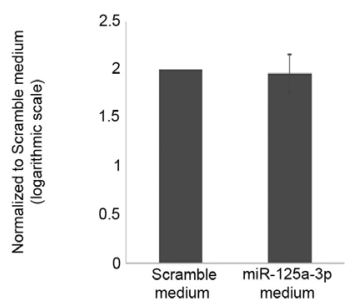

B miR-125a-3p expression in medium of transfected mural GCs

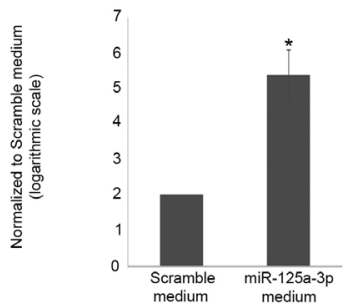

E Fyn mRNA expression in recipient cumulus cells

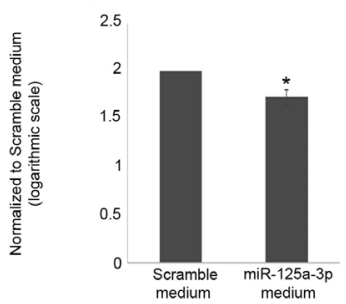

C miR-125a-3p expression in

recipient cumulus cells

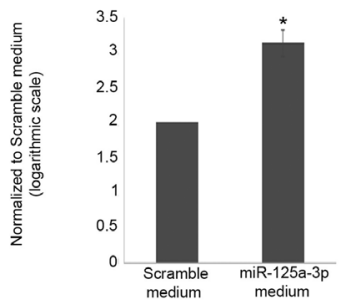

Figure 1 Mural GCs regulate the level of miR-125a-3p in CCs via the culture medium. Mural GCs were transfected with either scramble-miR or miR-125a-3p-mimic. Culture media of transfected cells were replaced after $4 \mathrm{~h}$ and cells were incubated for additional $16 \mathrm{~h}$. Transfected mural GCs were lysed, RNA was extracted and subjected to qPCR analysis for detection of miR-125a-3p. (A, B, and C) Normalized to the levels of the spiked-in cel-miR-39-3p (A). Culture media of mural GCs transfected with scramble-miR (scramble medium) or miR-125a-3p mimic (miR125a-3p medium) were collected and centrifuged. RNA was extracted from a portion of the medium and subjected to qPCR analysis for detection of miR-125a-3p (B). Freshly isolated CCs (recipient CCs) were seeded into the remaining conditioned media of transfected mural GCs and incubated overnight. Recipient CCs were lysed, RNA was extracted and subjected to qPCR analysis for the detection of miR-125a-3p (C), of pri-miR-125a normalized to the expression of U6 snRNA (D), and of Fyn mRNA (downstream target of miR-125a-3p), normalized to the expression of HPRT1 (E). Results are normalized to the values of their matching controls (scramble mural GCs; scramble CCs; scramble mural GCs/CCs medium). Log transformation was applied to mRNA levels. Data were analyzed by one-tail one-sample Student $t$-test. Bars are mean \pm S.E.M. of at least three independent experiments. ${ }^{*} P<0.05$.

\section{miR-125a-3p secreted by CCs can be taken up by mural GCs}

The well-established concept of bidirectional communication between mural GCs and CCs motivated us to examine the ability of CCs to regulate the level of miR-125a-3p within mural GCs. CCs transfected with miR-125a-3p mimic and thoroughly washed exhibited a significant over-expression of miR-125a-3p than CCs transfected with scrambled miR (Fig. 2A). Freshly isolated mural GCs incubated overnight in conditioned medium of transfected CCs exhibited a much higher level of miR-125a-3p expression when exposed to conditioned media of CCs transfected with miR-125a-3p mimic than those exposed to conditioned media of cells transfected with scrambled miR (Fig. 2B). This suggests that miR$125 a-3 p$, secreted by CCs into the culture medium, can be taken up by mural GCs.

\section{hCG regulates the expression level of miR-125a-3p in GCs and CCs}

Although miR-125a-3p is one of the miRNAs found in human FFs, neither its cellular source nor its possible role within the FFs have been described. In a previous study, we showed that hCG decreases the level of
miR-125a-3p expression in mural GCs (Grossman et al. 2015); it downregulates the secretion of miR-125a-3p by mural GCs, thus indirectly affecting CCs. hCG may also act, though to a lesser extent, directly on CCs, which express low levels of LHCGR (Jeppesen et al. 2012). A 6-h incubation of CCs with hCG resulted in a $30 \%$ decrease in the expression of miR-125-3p level (Fig. 2C). We found no change in the expression of miR$125 a-3 p$ within CCs after $2 \mathrm{~h}$ of incubation with hCG (data not shown). These findings indicate that hCG can directly downregulate the expression of miR-125a-3p within CCs in addition to the indirect effect exerted via mural GCs.

\section{Pri-miR-125a and miR-125a-3p accumulate within the oocyte throughout oogenesis}

Because fully grown GV oocytes are transcriptionally quiescent, we examined whether miR-125a-3p is transcribed and accumulated throughout oogenesis. Growing $(\leq 50 \mu \mathrm{m}$ diameter) and fully-grown (70-75 $\mu \mathrm{m}$ diameter) GV oocytes were isolated from 10-12 and 24-day-old mice, respectively, and stained to visualize their chromatin configuration, which indicates the state of their transcription activity that is correlated with their 
A miR-125a-3p expression in transfected cumulus cells

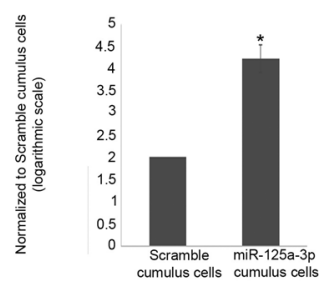

C miR-125a-3p expression in the medium of mural GCs after 2 hours incubation

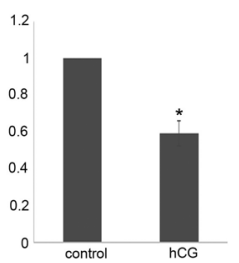

B miR-125a-3p expression in recipient mural GCs

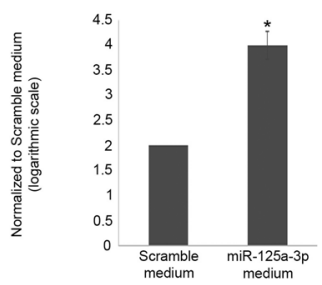

D miR-125a-3p expression in cumulus cells after 6 hours incubation

1.2

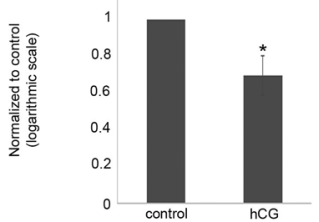

Figure 2 (A and B) Cumulus cells (CCs) regulate the level of miR-125a-3p in mural granulosa cells (GCs) via the culture medium. CCs were transfected with either scramble-miR or miR-125a-3pmimic. Culture media of transfected cells were replaced after $4 \mathrm{~h}$ and cells were incubated for additional $16 \mathrm{~h}$. Transfected CCs were washed, lysed, RNA was extracted and subjected to qPCR analysis for detection of miR-125a-3p, normalized to the levels of the spiked-in cel-miR-39-3p (A). Culture media of CCs transfected with scramble-miR ('scramble medium') or miR-125a-3p mimic ('miR$125 \mathrm{a}-3 \mathrm{p}$ medium') were collected and centrifuged. Freshly isolated mural GCs ('recipient mural GCs') were seeded into the conditioned media of transfected CCs and incubated overnight. Recipient mural GCs were lysed, RNA was extracted and subjected to qPCR analysis for the detection of miR-125a-3p (B). (C) hCG regulates the expression level of miR-125a-3p in CCs. CCs were incubated in the presence of $1 \mathrm{IU} / \mathrm{mL}$ hCG (hCG) or in saline (control). Cells were lysed and subjected to RNA extraction and qPCR analysis for the detection of miR-125a-3p, normalized to the level of U6-snRNA. Results are normalized to the values of control cells. Log transformation was applied to normalize mRNA levels. Data were analyzed by one-tail one-sample Student $t$-test. Bars are mean \pm S.E.M. of at least three independent experiments. ${ }^{*} P<0.05$.

size. Oocytes isolated from 10-12-day-old mice exhibited a de-condensed chromatin (non-surrounded nucleolus; NSN), confirming that they are growing oocytes capable of transcribing (Fig. 3 1, 1'; Bouniol-Baly et al. 1999). The fully grown GV oocytes, isolated from 24-day-old mice had condensed chromatin (surrounded nucleolus; SN), indicating their transcriptionally quiescent stage (BouniolBaly et al. 1999; Fig. 32 2' ). As stated earlier, fully grown oocytes are transcriptionally quiescent; therefore, it stands to reason that the pri-miR-125a is transcribed by growing oocytes and accumulates throughout oogenesis (Fig. 4). Though the level of miR-125a-5p was significantly higher than that of miR-125a-3p, in both growing and fully grown GV oocytes, it decreased during oogenesis, whereas the relative level of miR-125a-3p increased ( 2.5-folds; Fig. 4), implying a role for miR-125a-3p in fully grown GV oocytes.

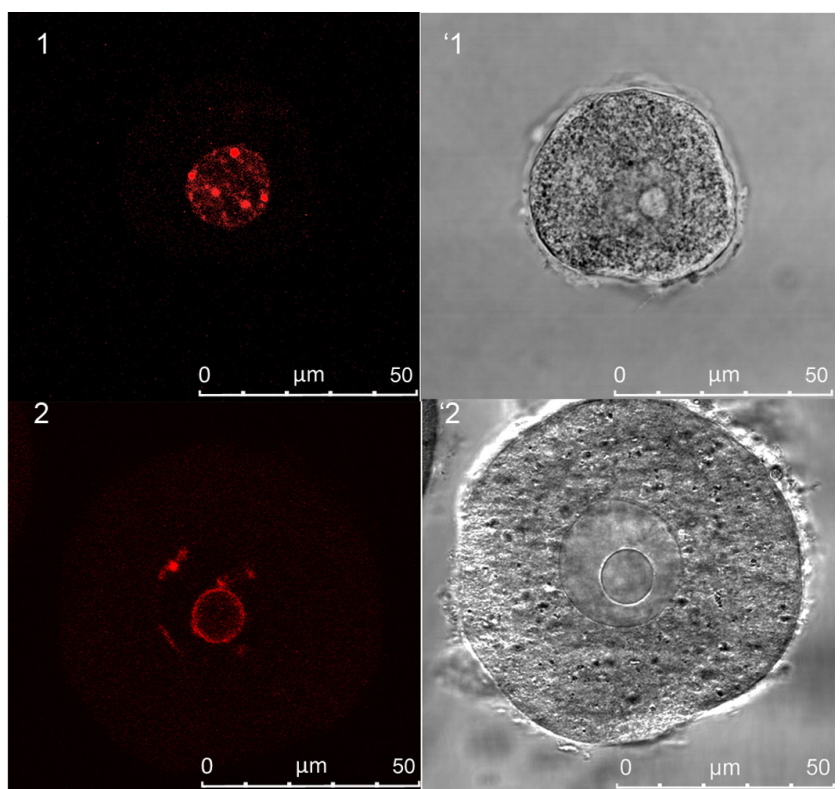

Figure 3 Pri-miR-125a and miR-125a-3p accumulate throughout oogenesis. Transcribing and fully grown GV oocytes were fixed and stained to confirm their NSN (1, $1^{\prime}$; transcribing GV oocyte) and SN $\left(2,2^{\prime}\right.$; fully-grown GV oocyte) heterochromatin configuration, which is correlated with their transcribing activity. $(1,2)$ DNA, labeled with Hoechst (red); (1', 2') bright field.

\section{hCG regulates pri-miR-125a, miR-125a-3p and miR- 125a-5p level within the enclosed oocytes}

Having established that hCG can regulate the expression of miR-125a-3p in the CCs, both directly and indirectly, we examined what kind of regulation operates in oocytes. The intercellular communications within the follicle, including those between mural GCs and CCs, as well as those between CCs and oocytes, are mediated mainly, but not solely, via gap junctions. We used an ex vivo model of cultured pre-ovulatory follicles, in which we initially exposed the follicles to hCG and validated that under our culture conditions, the oocytes maintain their ability to resume the first meiotic division (data not shown). We then examined the level of miR$125-3 p$ in oocytes isolated from follicles exposed to hCG and found that it decreased significantly compared to control oocytes (Fig. 5A). To examine whether CCs regulate the expression of miR-125a-3p in the oocytes via gap junctions alone, we added CBX to the culture media. The rationale behind the experimental design was to examine the effect of gap junction closure, which occurs when maturation is initiated, on the level of the miR-125a-3p and miR-125a-5p isoforms. However, as hCG induces closure of gap junction along with GVBD, we had to dissociate between the two events. We thus used CBX along with milrinone to determine the effect of closure of gap junctions, while the oocyte stayed at the GV stage. We then examined hCC treatment along 


\begin{tabular}{|c|c|c|c|}
\hline & Transcribing & Fully-grown & t-test \\
\hline pri-miR-125a & 0.59 & 3.46 & 0.050 \\
\hline miR-125a-5p & 94.35 & 84.51 & 0.020 \\
\hline miR-125a-3p & 5.06 & 12.03 & 0.050 \\
\hline
\end{tabular}

Transcribing

Fully-grown

pri-miR-125a

$\square \operatorname{miR}-125 a-5 p$

$\square$ miR-125a-3p
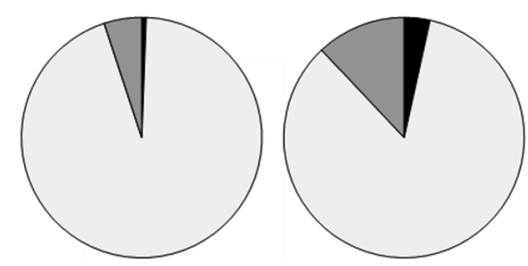

Figure 4 Changes in miR-125a family members. Transcribing and fully grown GV oocytes (ten oocytes of each) were lysed, RNA was extracted and subjected to qPCR analysis for the detection of miR-125a family members. Results present the percentage of each family member. Values are mean \pm S.E.M. of three independent experiments. ${ }^{*} P<0.05$. Data were analyzed by one-tail one-sample Student $t$-test.

with the milrinone on closure of the gap junctions by hCG, whereas the oocyte remained at the GV.

Our observation revealed that the presence of CBX resulted in an increased expression of miR-125a-3p and miR-125a-5p (Fig. 5B and C), implying that CCs contribute to the regulation of miR-125a-3p expression within the oocytes. Because oocytes in pre-ovulatory follicles are transcriptionally quiescent, the rise in miR$125 a-3 p$ expression cannot be explained by increased transcription. We showed that the level of pri-miR$125 \mathrm{a}$ in oocytes from CBX-treated follicles decreased by $\sim 60 \%$ compared to that in oocytes from untreated follicles (Fig. 5D). These findings suggest that the increase in the expression of miR-125-3p is, at least partially, due to increased processing of pri-miR-125a, rather than to inhibition of degradation, or alternatively, to transfer of miR-125a-3p between the oocyte and its surrounding CCs.

\section{Discussion}

The current study addresses the subject of pre-ovulatory intra-follicular intercellular regulation of miR-125a-3p. Our findings indicate that the expression of miR-125a-3p within mural GCs, CCs, and oocytes is regulated through the FF and via intercellular communication by a net of gap-junctions. Moreover, we demonstrated that hCG can downregulate, both directly and indirectly, the preovulatory expression of miR-125a-3p within the follicle.

The presence of circulating miRNAs in body fluids is well established, with the assumption that they serve as a
A miR-125a-3p expression in oocytes of hCG treated large follicles

miR-125a-3p expression in oocytes 1.2

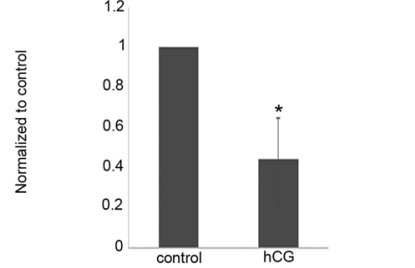
of CBX treated large follicles

C miR-125a-5p expression in oocytes of $\mathrm{CBX}$ treated large follicles

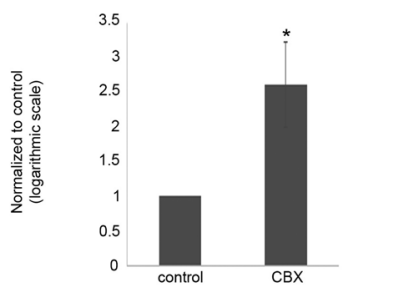

D pri-miR-125a expression in oocytes of CBX treated large follicles
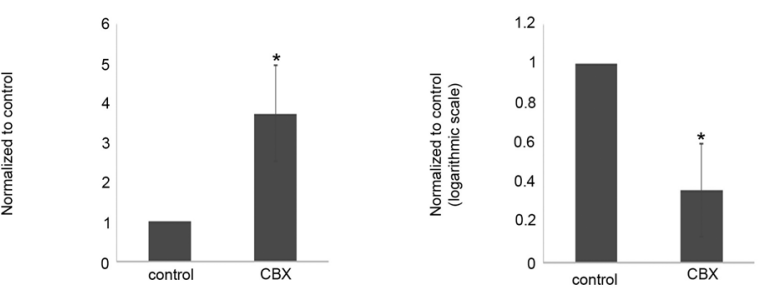

Figure 5 hCG regulates the levels of pri-miR-125a, miR-125a-3p and miR-125a-5p within oocytes. Large antral follicles were incubated at $37^{\circ} \mathrm{C}$ in constant shaking with $1 \mathrm{IU} / \mathrm{mL}$ hCG $(\mathrm{A})$, gap-junctions inhibitor (CBX, $100 \mu \mathrm{M}$; B, C, and D) or with an equal volume of vehicle (control; $A, B, C$, and D); all in the presence of milrinone. Oocytes (30 in each treatment group) were isolated, lysed, and their RNA was extracted and subjected to qPCR analysis for the detection of miR-125a-3p (A and B), miR-125a-5p (C), and pri-miR-125a (D). Results were normalized to the values of control oocytes. Data were analyzed by one-tail one-sample Student $t$-test. Bars are mean \pm S.E.M. of at least three independent experiments. ${ }^{*} P<0.05$.

means to pass information among cells. Most circulating miRNAs are contained within extracellular vesicles, whereas others are bound to proteins that protect them from being degraded (Mittelbrunn \& Sánchez-Madrid 2012, Machtinger et al. 2016). Once released, the ability of circulating miRNAs to target specific cells is limited. Though there is some evidence for a cellspecific internalization of extracellular vesicles (Raposo \& Stoorvogel 2013), the inevitable dilution of circulating miRNAs in body fluids means that an efficient transfer is restricted to adjacent cells (Lemcke et al. 2015). Several miRNAs have been detected in human, mare, and bovine FFs (da Silveira et al. 2015, Di Pietro 2016). miR125a-3p was detected in human FF (Sang et al. 2013). In the current study we demonstrated that mouse mural GCs can secrete miR-125a-3p; the secreted quantity depends on the level of miR-125a-3p within the cells. This finding is in accordance with a recent study, which reported that the level of exosomal miR-222 in the culture media reflects its abundance in the cells of origin (Felicetti et al. 2016). Nevertheless, it should be considered that different outcomes might be achieved using naive, rather than transfected, cells. While the expression of miR-125a-3p increased in CCs incubated in conditioned medium of miR-125a-3p over-expressing mural GCs, the level of nuclear pri-miR-125a remained 
constant; suggesting that the increase in miR-125a-3p within the cells is due to uptake from the culture medium rather than to increased processing of pri-miR-125a. The increase in miR-125a-3p in the culture medium was not caused by its release from dead cells, as verified by an XTT viability test. However, we cannot exclude the possibility that at least some of the increase in miR$125 \mathrm{a}-3 \mathrm{p}$ expression is the result of altered processing of pre-miR-125a or of reduced degradation. In accordance with the observation that transferred miRNAs remain functional within recipient cells and modulate their function, we could detect a downregulation of Fyn mRNA in recipient CCs, indicating that the miR$125 a-3 p$ retained its ability to influence gene expression within recipient CCs. Although we found a vast increase in miR-125a-3p expression in CCs, there was a minor decrease in Fyn mRNA, implying that Fyn expression is also regulated by other control mechanisms.

A complementary reverse experiment showed an increase in miR-125a-3p expression within mural GCs incubated in conditioned medium of miR-125a-3pover-expressing CCs, implying transfer of miR-125a-3p itself. In summary, the presence of miR-125a-3p in the $F F$, the two reciprocal experiments, and the observation that the miR-125a-3p is functional in the recipient cells, imply that regulation of miR-125a-3p expression in GCs and CCs is bidirectional and mediated through the FF. Accumulation of miR-125a-3p was not expected, because of the shorthalf-life of miRNAs in culture medium supplemented with FCS (which contains RNAses).

hCG directly downregulates the expression of miR$125 a-3 p$ in CCs, as evidenced by the $30 \%$ decrease in the expression of miR-125a-3p, after $6 \mathrm{~h}$ of incubation with hCG. In a previous study we showed that hCG downregulates the expression of miR-125a-3p within mural GCs toward ovulation, so that the decreased expression enables increase in Fyn expression, cell migration, and proper ovulation (Grossman et al. 2015). Migration of CCs occurs in response to ovulatory doses of hCG and is assumed to participate in the process of CCs expansion (Akison et al. 2012). Hence, it is possible that the direct- and indirect-mediated decrease in miR$125 \mathrm{a}-3 \mathrm{p}$ within CCs is necessary to allow CCs expansion. miRNA can be transported between cells via cell-cell structures such as synapses and gap-junctions as well as by extracellular vesicles (Mittelbrunn \& SánchezMadrid 2012, Machtinger et al. 2016). miRNAs were suggested to be transported between cells through gap junctions in a selective manner (Aucher et al. 2013). The type of connexins forming the gap junctions contribute to their selectivity, whereas other connexins possess diverse abilities to transfer miRNAs (Valiunas et al. 2005, Zong et al. 2016). When examining intercellular communications within the follicle, it is necessary to confirm or refute a possible interactions mediated through the well-established net of gap junctions between oocytes and CCs. Incubating large antral follicles with the gap-junction inhibitor, CBX, resulted in an increase in the expression of miR-125a-3p within the enclosed oocyte. Because fully grown GV oocytes are transcriptionally quiescent (Zuccotti et al. 2011), the increase in miR-125a-3p expression is not a result of increased transcription but rather a result of either inhibited degradation or increased processing of the primiR-125a. To differentiate between the two possibilities, we monitored the expression of pri-miR-125a and miR-125a-5p. CBX caused an increase in miR-125a-5p expression, while causing a decrease in that of pri-miR125a. Thus, we assume that at least part of the increase in miR-125a-3p in response to gap-junctions closure is caused by accelerated processing of the pri-miR-125a to produce the mature forms, miR-125a-3p and miR$125 \mathrm{a}-5 \mathrm{p}$. Theoretically, because gap junctions permit bidirectional communications within the cumulusoocyte complex (Kidder \& Vanderhyden 2010), the oocyte could transport miR-125a-3p and miR-125a-5p to the CCs; if the gap junctions are closed, both miRNAs would accumulate in the oocyte. This option is less likely to occur as it does not support the observed decrease in the amount of pri-miR-125a. Our findings imply that CCs regulate, through the gap-junctions, the level of miR-125a-3p expression within the oocytes.

In a previous study we found that the decrease in the expression of oocyte miR-125a-3p, caused by hCG, was required for resumption of the first meiotic division (Grossman et al. 2017). Surprisingly, unlike in oocytes exposed to hCG in vivo, we observed an increase in the expression of miR-125a-3p within oocytes derived from CBX-treated follicles, instead of the expected decrease. This contradiction may be explained by the different modes of action of hCG and CBX. CBX blocks only gap junctions, whereas hCG activates several signaling pathways within the follicle, by activating the LHCGR (Stocco et al. 2007).

Transcription activity in fully grown GV oocytes ceases as the oocytes acquire the ability to resume first meiotic division, concomitantly with reconfiguration of the oocyte chromatin (De La Fuente et al. 2004). Transcription in mice oocytes is initiated only at the late zygote stage with activation of the embryonic genome. Thus, oocyte maturation, fertilization, and early embryonic development rely on pre-synthesized maternal RNA transcripts. Lack of transcription activity necessitates utilization of post-transcriptional regulation mechanisms to adjust the expression of mRNAs required for critical stages of development, including formation of RNA granules (Kotani et al. 2013), elongation of the poly-A tail of mRNA (Vassalli et al. 1989), and RNA silencing, which employs both siRNAs and miRNAs (Murchison et al. 2007, Kaneda et al. 2009). In contrast to siRNAs, the contribution of miRNAs to the wellness of fully grown GV oocytes is controversial. The results of a luciferase reporter assay, used to examine the activity of two highly abundant endogenous miRNAs 
A

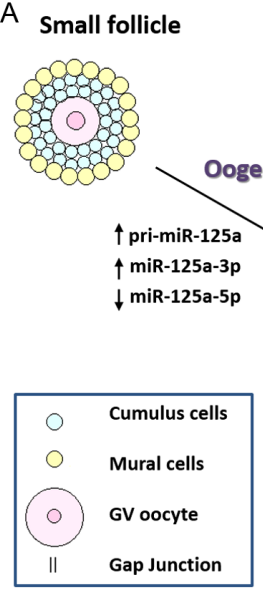

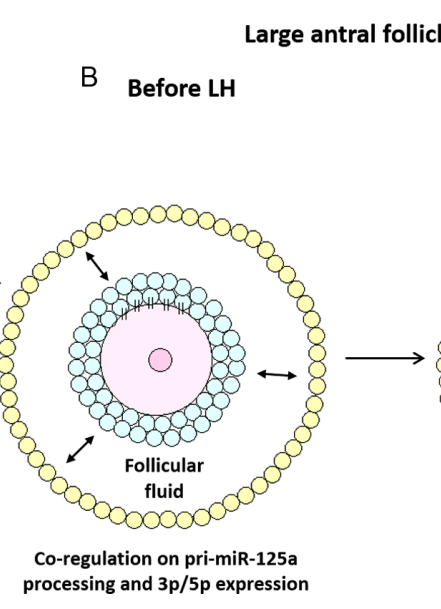

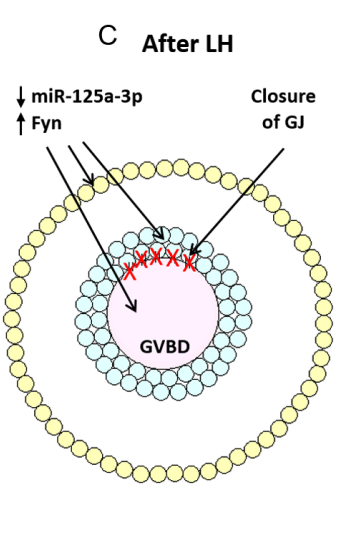

Figure 6 A schematic model of miR-125a-3p regulation within the ovarian follicles. Throughout oogenesis (A), pri-miR-125a is transcribed and accumulates in the growing oocyte. In both growing and fully grown GV oocytes, the level of miR-125a-5p is significantly higher than that of miR-125a-3p, however, it decreases during oogenesis, whereas the relative level of miR-125a-3p increases. In the large antral follicle (B), the expression of miR-125a-3p is intracellularly regulated in mural GCs, CCs, and oocytes. Though the cells are capable of transcribing miR-125a-3p to achieve the response to $\mathrm{LH}$, the decrease in miR-125a-3p expression is co-regulated by intercellular communications within the follicle, including the transfer of miR-125a-3p through the follicular fluid. Upon LH surge (C), the expression of miR-125a-3p decrease in a coordinated manner in each of these cell types, allowing increase of FYN, presumably to support the molecular events occurring towards ovulation. in oocytes, indicated that their activity is suppressed, in fully grown GV oocytes compared to growing oocytes, though not completely abolished (Ma et al. 2010). The decreased ability of miRNAs to inhibit the expression of the luciferase reporter suggests that distinct miRNAs might be subjected to different regulatory mechanisms to enable a time-restricted activity of miRNAs. The regulation, by hCG, of miR-125a-3p expression in oocytes suggests that it is one of the miRNAs that play a role in fully grown GV oocytes, a suggestion supported by our recent finding that miR-125a-3p participates in maintaining the first meiotic arrest by modulating the actin cytoskeleton (Grossman et al. 2017).

The transcriptionally quiescent state of the oocytes, along with our findings indicating that CCs probably do not transport miR-125a-3p to the maturing oocytes, suggest that miR-125a-3p within fully grown GV oocyte must have been already transcribed and accumulated within the growing oocytes. Accumulation of miRNAs throughout mouse oogenesis has been demonstrated by Tang et al. who showed that many, but not all, of the vast array of miRNAs expressed by the oocyte are preferably accumulated during oogenesis (Tang et al. 2007). Interestingly, although the expression level of miR-125a-5p was significantly higher than that of miR$125 a-3 p$ in the growing and fully grown GV oocytes, the relative amount of miR-125a-5p decreased during oogenesis whereas that of miR-125a-3p increased. This observation suggests that although miR-125a-3p is less abundant, the oocyte favors its accumulation over that of miR-125a-5p, a difference that can be attributed to alteration in the structure of the pre-miRNA, which can dictate strand preference (Park et al. 2011, StaregaRoslan et al. 2011). Our finding that the level of each of the mature miRNAs was significantly higher than that of pri-miR-125a is supported by a study indicating that for most examined miRNAs, the amount of mature miRNA was higher than the sum of the pre-miRNA and the primiRNA (Volk \& Shomron 2011).

In conclusion, the findings in this study, summarized in Fig. 6, indicate that pri-miR-125a is transcribed and accumulated in the growing oocyte during oogenesis. In the large antral follicle, the expression of miR-125a-3p is intracellularly regulated in mural GCs, CCs, and oocytes. Though the cells are capable of transcribing miR-125a-3p to achieve synchronized response to hCG, the decrease in miR-125a-3p expression is modulated by intercellular communications within the follicle, including the transfer of miR-125a-3p through the FF. Upon LH surge, the expression of miR-125a-3p decreases in a coordinated manner in each of these cell types, allowing the increase of FYN, presumably to support the molecular events occurring toward ovulation.

\section{Declaration of interest}

The authors declare that there is no conflict of interest that could be perceived as prejudicing the impartiality of the research reported.

\section{Funding}

This work was partially supported by a grant from the Israel Science Foundation (No. 470/14) to Ruth Shalgi.

\section{Author contribution statement}

H G developed the concept, wrote the paper, designed and carried out the experiments, organized the data, and performed the statistical analyses, along with E H-P who performed this work in partial fulfillment of the requirements for an M.Sc. 
degree. I M assisted in analyzing the data; R S conceived the study, participated in its design and coordination, helped draft the manuscript, and supervised the study. All authors read the final manuscript and approved it.

\section{Acknowledgments}

The authors are grateful to Ruth Kaplan-Kraicer from Sackler Faculty of Medicine, Tel Aviv University, for her help in preparing the manuscript.

\section{References}

Akison LK, Alvino ER, Dunning KR, Robker RL \& Russell DL 2012 Transient invasive migration in mouse cumulus oocyte complexes induced at ovulation by luteinizing hormone. Biology of Reproduction 86 1-8. (https://doi.org/10.1095/biolreprod.111.097345)

Aucher A, Rudnicka D \& Davis DM 2013 MicroRNAs transfer from human macrophages to hepato-carcinoma cells and inhibit proliferation. Journal of Immunology 191 6250-6260. (https://doi.org/10.4049/ jimmunol.1301728)

Bartel DP \& Chen CZ 2004 Micromanagers of gene expression: the potentially widespread influence of metazoan microRNAs. Nature Reviews: Genetics 5 396-400. (https://doi.org/10.1038/nrg1328)

Bouniol-Baly C, Hamraoui L, Guibert J, Beaujean N, Szöllösi MS \& Debey P 1999 Differential transcriptional activity associated with chromatin configuration in fully grown mouse germinal vesicle oocytes. Biology of Reproduction 60 580-587. (https://doi.org/10.1095/biolreprod60.3.580)

Canipari R, Cellini V \& Cecconi S 2012 The ovary feels fine when paracrine and autocrine networks cooperate with gonadotropins in the regulation of folliculogenesis. Current Pharmaceutical Design 18 245-255. (https:// doi.org/10.2174/138161212799040411)

Chen X, Ba Y, Ma L, Cai X, Yin Y, Wang K, Guo J, Zhang Y, Chen J, Guo X et al. 2008 Characterization of microRNAs in serum: a novel class of biomarkers for diagnosis of cancer and other diseases. Cell Research 18 997-1006. (https://doi.org/10.1038/cr.2008.282)

Cheng G 2015 Circulating miRNAs: roles in cancer diagnosis, prognosis and therapy. Advanced Drug Delivery Reviews 81 75-93. (https://doi. org/10.1016/j.addr.2014.09.001)

Conti M, Zamah AM, Franciosi F, Cakmak H \& Cedars MI 2016 Dynamic secretion during meiotic reentry integrates the function of the oocyte and cumulus cells. PNAS 113 2424-2429. (https://doi.org/10.1073/ pnas.1519990113)

da Silveira JC, Veeramachaneni DNR, Winger QA, Carnevale EM \& Bouma GJ 2012 Cell-secreted vesicles in equine ovarian follicular fluid contain miRNAs and proteins: a possible new form of cell communication Within the ovarian follicle. Biology of Reproduction $\mathbf{8 6}$ 71. (https://doi.org/10.1095/biolreprod.111.093252)

da Silveira JC, de Andrade GM, Nogueira MFG, Meirelles FV \& Perecin F 2015 Involvement of miRNAs and cell-secreted vesicles in mammalian ovarian antral follicle development. Reproductive Sciences 22 1474-1483. (https://doi.org/10.1177/1933719115574344)

De La Fuente R, Viveiros MM, Burns KH, Adashi EY, Matzuk MM \& Eppig JJ 2004 Major chromatin remodeling in the germinal vesicle (GV) of mammalian oocytes is dispensable for global transcriptional silencing but required for centromeric heterochromatin function. Developmental Biology 275 447-458. (https://doi.org/10.1016/j.ydbio.2004.08.028)

Di Pietro C 2016 Exosome-mediated communication in the ovarian follicle. Journal of Assisted Reproduction and Genetics 33 303-311. (https://doi.org/10.1007/s10815-016-0657-9)

Donadeu FX \& Schauer SN 2013 Differential miRNA expression between equine ovulatory and anovulatory follicles. Domestic Animal Endocrinology 45 122-125. (https://doi.org/10.1016/j. domaniend.2013.06.006)

Felicetti F, De Feo A, Coscia C, Puglisi R, Pedini F, Pasquini L, Bellenghi M, Errico MC, Pagani E \& Carè A 2016 Exosome-mediated transfer of miR222 is sufficient to increase tumor malignancy in melanoma. Journal of Translational Medicine 14 56. (https://doi.org/10.1186/s12967-0160811-2)
Grossman H, Chuderland D, Ninio-Many L, Hasky N, Kaplan-Kraicer R \& Shalgi R 2015 A novel regulatory pathway in granulosa cells, the LH/human chorionic gonadotropin-microRNA-125a-3p-Fyn pathway, is required for ovulation. FASEB Journal 29 3206-3216. (https://doi. org/10.1096/fj.14-269449)

Grossman H, Har-Paz E, Gindi N, Levi M, Miller I, Nevo N, Galiani D, Dekel N \& Shalgi R 2017 Regulation of GVBD in mouse oocytes by miR$125 \mathrm{a}-3 \mathrm{p}$ and Fyn kinase through modulation of actin filaments. Scientific Reports 7 2238. (https://doi.org/10.1038/s41598-017-02071-x)

Hata T, Murakami K, Nakatani H, Yamamoto Y, Matsuda T \& Aoki N 2010 Isolation of bovine milk-derived microvesicles carrying mRNAs and microRNAs. Biochemical and Biophysical Research Communications 396 528-533. (https://doi.org/10.1016/j.bbrc.2010.04.135)

Heller DT, Cahill DM \& Schultz RM 1981 Biochemical studies of mammalian oogenesis: metabolic cooperativity between granulosa cells and growing mouse oocytes. Developmental Biology 84 455-464. (https://doi.org/10.1016/0012-1606(81)90415-2)

Jeppesen JV, Kristensen SG, Nielsen ME, Humaidan P, Dal Canto M, Fadini R, Schmidt KT, Ernst E \& Andersen CY 2012 LH-receptor gene expression in human granulosa and cumulus cells from antral and preovulatory follicles. Journal of Clinical Endocrinology and Metabolism 97 1524-1531. (https://doi.org/10.1210/jc.2012-1427)

Kaneda M, Tang F, O'Carroll D, Lao K \& Surani MA 2009 Essential role for Argonaute2 protein in mouse oogenesis. Epigenetics and Chromatin 29. (https://doi.org/10.1186/1756-8935-2-9)

Kidder GM \& Mhawi AA 2002 Gap junctions and ovarian folliculogenesis. Reproduction 123 613-620. (https://doi.org/10.1530/rep.0.1230613)

Kidder GM \& Vanderhyden BC 2010 Bidirectional communication between oocytes and follicle cells: ensuring oocyte developmental competence. Canadian Journal of Physiology and Pharmacology 88 399-413. (https:// doi.org/10.1139/y10-009)

Komatsu K \& Masubuchi S 2018 Mouse oocytes connect with granulosa cells by fusing with cell membranes and form a large complex during follicle development. Biology of Reproduction 94 19-22. (https://doi. org/10.1093/biolre/ioy072)

Kotani T, Yasuda K, Ota R \& Yamashita M 2013 Cyclin B1 mRNA translation is temporally controlled through formation and disassembly of RNA granules. Journal of Cell Biology 202 1041-1055. (https://doi. org/10.1083/jcb.201302139)

Lemcke H, Steinhoff G \& David R 2015 Gap junctional shuttling of miRNA - a novel pathway of intercellular gene regulation and its prospects in clinical application. Cellular Signalling 27 2506-2514. (https://doi. org/10.1016/j.cellsig.2015.09.012)

Levi M, Maro B \& Shalgi R 2010a The involvement of Fyn kinase in resumption of the first meiotic division in mouse oocytes. Cell Cycle $\mathbf{9}$ 1577-1589. (https://doi.org/10.4161/cc.9.8.11299)

Levi M, Maro B \& Shalgi R 2010b Fyn kinase is involved in cleavage furrow ingression during meiosis and mitosis. Reproduction 140 827-834. (https://doi.org/10.1530/REP-10-0312)

Lorenzen JM, Volkmann I, Fiedler J, Schmidt M, Scheffner I, Haller H, Gwinner W \& Thum T 2011 Urinary miR-210 as a mediator of acute T-cell mediated rejection in renal allograft recipients. American Journal of Transplantation 11 2221-2227. (https://doi.org/10.1111/j.16006143.2011.03679.x)

Ma J, Flemr M, Stein P, Berninger P, Malik R, Zavolan M, Svoboda P \& Schultz RM 2010 MicroRNA activity is suppressed in mouse oocytes. Current Biology 20 265-270. (https://doi.org/10.1016/j.cub.2009.12.042)

Ma JY, Li M, Luo YB, Song S, Tian D, Yang J, Zhang B, Hou Y, Schatten H, Liu Z et al. 2013 Maternal factors required for oocyte developmental competence in mice: transcriptome analysis of non-surrounded nucleolus (NSN) and surrounded nucleolus (SN) oocytes. Cell Cycle 12 1928-1938. (https://doi.org/10.4161/cc.24991)

Machtinger R, Laurent LC \& Baccarelli AA 2016 Extracellular vesicles: roles in gamete maturation, fertilization and embryo implantation. Human Reproduction Update 22 182-193. (https://doi.org/10.1093/ humupd/dmv055)

Michael A, Bajracharya SD, Yuen PST, Zhou H, Star RA, Illei GG \& Alevizos I 2010 Exosomes from human saliva as a source of microRNA biomarkers. Oral Diseases 16 34-38. (https://doi.org/10.1111/j.16010825.2009.01604.x)

Mittelbrunn M \& Sánchez-Madrid F 2012 Intercellular communication: diverse structures for exchange of genetic information. Nature Reviews: Molecular Cell Biology 13 328-335. (https://doi.org/10.1038/nrm3335) 
Moreno JM, Núñez MJ, Quiñonero A, Martínez S, De La Orden M Simón C, Pellicer A, Díaz-García C \& Domínguez F 2015 Follicular fluid and mural granulosa cells microRNA profiles vary in in vitro fertilization patients depending on their age and oocyte maturation stage. Fertility and Sterility 104 1037.e1-1046.e1. (https://doi.org/10.1016/j. fertnstert.2015.07.001)

Murchison EP, Stein P, Xuan Z, Pan H, Zhang MQ, Schultz RM \& Hannon GJ 2007 Critical roles for Dicer in the female germline. Genes and Development 21 682-693. (https://doi.org/10.1101/gad.1521307)

Ninio-Many L, Grossman H, Shomron N, Chuderland D \& Shalgi R 2013 microRNA-125a-3p reduces cell proliferation and migration by targeting Fyn. Journal of Cell Science 126 2867-2876. (https://doi.org/10.1242/ jcs.123414)

Noferesti SS, Sohel MMH, Hoelker M, Salilew-Wondim D, Tholen E, Looft C, Rings F, Neuhoff C, Schellander K \& Tesfaye D 2015 Controlled ovarian hyperstimulation induced changes in the expression of circulatory miRNA in bovine follicular fluid and blood plasma. Journal of Ovarian Research 8 81. (https://doi.org/10.1186/s13048-015-0208-5)

Park JE, Heo I, Tian Y, Simanshu DK, Chang H, Jee D, Patel DJ \& Kim VN 2011 Dicer recognizes the $5^{\prime}$ end of RNA for efficient and accurate processing. Nature 475 201-205. (https://doi.org/10.1038/nature10198)

Pepling ME 2012 Follicular assembly: mechanisms of action. Reproduction 143 139-149. (https://doi.org/10.1530/REP-11-0299)

Raposo G \& Stoorvogel W 2013 Extracellular vesicles: exosomes, microvesicles, and friends. Journal of Cell Biology 200 373-383. (https:// doi.org/10.1083/jcb.201211138)

Sang Q, Yao Z, Wang HH, Feng R, Wang HH, Zhao X, Xing Q, Jin L, He L, Wu L et al. 2013 Identification of microRNAs in human follicular fluid: characterization of microRNAs that govern steroidogenesis in vitro and are associated with polycystic ovary syndrome in vivo. Journal of Clinical Endocrinology and Metabolism 98 3068-3079. (https://doi.org/10.1210/ jc.2013-1715)

Sela-Abramovich S, Edry I, Galiani D, Nevo N \& Dekel N 2006 Disruption of gap junctional communication within the ovarian follicle induces oocyte maturation. Endocrinology 147 2280-2286. (https://doi. org/10.1210/en.2005-1011)

Smitz JEJ \& Cortvrindt RG 2002 The earliest stages of folliculogenesis in vitro. Reproduction 123 185-202. (https://doi.org/10.1530/rep.0.1230185)

Starega-Roslan J, Koscianska E, Kozlowski P \& Krzyzosiak WJ 2011 The role of the precursor structure in the biogenesis of microRNA. Cellular and Molecular Life Sciences 68 2859-2871. (https://doi.org/10.1007/ s00018-011-0726-2)

Stocco C, Telleria C \& Gibori G 2007 The molecular control of corpus luteum formation, function, and regression. Endocrine Reviews 28 117-149. (https://doi.org/10.1210/er.2006-0022)

Su H, Trombly MI, Chen J \& Wang X 2009 Essential and overlapping functions for mammalian Argonautes in microRNA silencing. Genes and Development 23 304-317. (https://doi.org/10.1101/gad.1749809)
Sugiura K, Su YQ, Li Q, Wigglesworth K, Matzuk MM \& Eppig JJ 2010 Estrogen promotes the development of mouse cumulus cells in coordination with oocyte-derived GDF9 and BMP15. Molecular Endocrinology 24 2303-2314. (https://doi.org/10.1210/me.2010-0260)

Sun QY, Miao YL \& Schatten H 2009 Towards a new understanding on the regulation of mammalian oocyte meiosis resumption. Cell Cycle $\mathbf{8}$ 2741-2747. (https://doi.org/10.4161/cc.8.17.9471)

Tan JH, Wang HL, Sun XS, Liu Y, Sui HS \& Zhang J 2009 Chromatin configurations in the germinal vesicle of mammalian oocytes. Molecular Human Reproduction 15 1-9. (https://doi.org/10.1093/molehr/gan069)

Tang F, Kaneda M, O'Carroll D, Hajkova P, Barton SC, Sun YA, Lee C, Tarakhovsky A, Lao K \& Surani MA 2007 Maternal microRNAs are essential for mouse zygotic development. Genes and Development 21 644-648. (https://doi.org/10.1101/gad.418707)

Valiunas V, Polosina YY, Miller H, Potapova IA, Valiuniene L, Doronin S, Mathias RT, Robinson RB, Rosen MR, Cohen IS et al. 2005 Connexinspecific cell-to-cell transfer of short interfering RNA by gap junctions. Journal of Physiology $\mathbf{5 6 8}$ 459-468. (https://doi.org/10.1113/ jphysiol.2005.090985)

Vassalli JD, Huarte J, Belin D, Gubler P, Vassalli A, O'Connell ML, Parton LA, Rickles RJ \& Strickland S 1989 Regulated polyadenylation controls mRNA translation during meiotic maturation of mouse oocytes. Genes and Development 3 2163-2171. (https://doi.org/10.1101/ gad.3.12b.2163)

Volk N \& Shomron N 2011 Versatility of microRNA biogenesis. PLoS ONE 6 e19391. (https://doi.org/10.1371/journal.pone.0019391)

Yang CR, Lowther KM, Lalioti MD \& Seli E 2016 Embryonic poly(A)binding protein (EPAB) is required for granulosa cell EGF signaling and cumulus expansion in female mice. Endocrinology 157 405-416. (https://doi.org/10.1210/en.2015-1135)

Zong L, Zhu Y, Liang R \& Zhao HB 2016 Gap junction mediated miRNA intercellular transfer and gene regulation: a novel mechanism for intercellular genetic communication. Scientific Reports 6 19884. (https:// doi.org/10.1038/srep19884)

Zuccotti M, Bellone M, Longo F, Redi CA \& Garagna S 2011 Fully-mature antral mouse oocytes are transcriptionally silent but their heterochromatin maintains a transcriptional permissive histone acetylation profile. Journal of Assisted Reproduction and Genetics 28 1193-1196. (https:// doi.org/10.1007/s10815-011-9562-4)

Received 3 September 2019

First decision 30 September 2019

Revised manuscript received 10 December 2019

Accepted 16 December 2019 